\title{
OBSERVATIONAL PARAMETERS: DEFINITIONS AND LIMITS
}

\author{
J.H. LUTZ \\ Program in Astronomy, Washington State University, Pullman, Washington USA
}

This review paper gives an overview of many of the observational topics that will be covered during the symposium. Trends and new developments in planetary nebula observations will be highlighted.

\section{Introduction}

The definition of a "planetary nebula" (PN) is clear enough as long as the terms are kept fairly general. A PN is a star that ejects some material while evolving from the red giant to the white dwarf stage. However, when we try to get more specific about particular sub-phases of the evolution, different observers adopt quite diverse terminologies. The following adjectives have been used to describe groups of planetary nebulae in the recent literature: proto-planetary nebulae, young preplanetary nebulae, young PN, compact PN, evolved PN, large PN and higherly evolved PN. Presumably this list is in some rough order from the beginning to the end of the PN stage, but this is not entirely clear.

Further complicating the matter are several types of objects that are thought to be related, at least in part, to the PN stage. These include OH/IR stars, some IRAS sources, Mira variable stars, symbiotic stars, and calaclysmic variables. All but the cataclysmic variables are associated with the early stages of PN evolution.

Observational questions such as "what objects are representative of the earliest stage at which we can detect the nebula" will be the subject of much debate during this conference. Also, the extent to which objects such as $\mathrm{OH} / \mathrm{IR}$ stars represent a stage in the evolution of all PN remains to be studied.

We are in a fascinating time with regard to the observations of PN. New hardware such as CCD's, IR detectors and space-borne telescopes have greatly expanded the capacity to obtain data. On the other hand, some fundamental data (such as distances) remain surprisingly elusive.

\section{How Many and Where?}

PN are spreading out. The number quoted for scale height in textbooks and previous symposia is about 150 pc. However, Zilstra and Pottasch (1991) find that the scale height is somewhat larger: $250 \pm 50 \mathrm{pc}$ assuming an exponential model of the galactic disk or $190 \pm 40 \mathrm{pc}$ assuming an isothermal disk. Their estimate for the total number of $\mathrm{PN}$ in the disk is $23,000 \pm 6000$.

Accurate distances to large numbers of individual PN remain elusive. Most studies continue to rely upon the relatively crude statistical methods. However, a new application of a method that was used a number of years ago to find distances to individual PN from optical images shows great promise. This is the expansion parallax as measured from radio maps obtained at different epochs. Masson (1989) used VLA maps from three different epochs of observation to calculate a distance of

R. Weinberger and A. Acker (eds.), Planetary Nebulae, 19-22.

(C) 1993 IAU. Printed in the Netherlands. 
$880 \pm 150$ pc for NGC 7027. Seaquist (1991) was able to put a limit on the distance of $V y 2-2$ by measuring the radio expansion parallax.

\section{Temperatures, Densities, Abundances}

CCD's have made possible a vast expansion of the databases on PN physical parameters. Still, the typical PN with visible shells have electron temperatures of about $1-2 \times 10^{4} \mathrm{~K}$ and electron densities of about $10^{2}-10^{7} \mathrm{~cm}^{-3}$.

Authors tend to confine their studies to a particular physical parameter or to some identifiable subset of PN. For example, Stanghellini and Kaler (1989) studied electron densities derived from [O II], [Cl III], [S II] and [Ar IV] in $146 \mathrm{PN}$. Rowlands (1989) investigated electron temperatures in the high excitation zones of $\mathrm{PN}$ by using [Ne V] lines. Kaler (1990) concentrated on the characteristics of 75 large PN. Henry (1990) included 192 PN from the Milky Way Galaxy, the LMC, the SMC and M31 in a comprehensive study of abundance patterns. He concluded that nitrogen is produced via different thermonuclear cycles in different groups of PN.

Connected intimately to the determination of physical parameters is the issue of whether a particular PN is density bounded or ionization bounded. Zahng and Kwok (1991) analyzed spectra from 0.1 to 100 microns for 66 compact PN and concluded that about $90 \%$ of them were ionization bounded. Further pursuit of their broad-based approach will lead to progress on the issue of whether or not most PN have neutral material surrounding the ionized regions.

Studies of PN in the galactic bulge have addressed a variety of topics Webster (1988) provided the first large scale investigation of the masses and abundances of bulge PN. Most recently Ratag, et al (1992) studied 120 bulge PN. Dopita, et al (1990) did high resolution imaging of bulge $\mathrm{PN}$, achieving a resolution 0.35 arcsec by using photon counting and image reconstruction techniques.

A review of the considerable literature that has accumulated recently on extragalactic PN will be left to those speaking on the subject later in the conference.

\section{Molecules, Infrared, Dust}

At previous IAU symposia on PN, studies of molecules were scarce and the number of objects detected was low. What a change we see now! In particular, there has been an extension of the CO surveys such that detections are occurring frequently. Huggins and Healey (1989) surveyed $100 \mathrm{PN}$ and detected CO in 19 of them. It is now possible to estimate both the molecular mass and the ionized mass of a nebula, and sometimes the molecular mass is considerably larger. For example, Gomez, et al (1992) found that the molecular mass of the young PN M3-28 is 0.2 solar masses, whereas the ionized mass is 0.03 solar masses. In evolved PN the ionized mass dominates (Healey and Huggins 1990).

Other molecular studies include the discovery of ammonia in the proto-planetary nebulae CRL618 and CRL2688 (Martin-Pintado and Bachiller 1992). Many more detections can be expected as millimeter and infrared detectors improve. 
In the infrared, IRAS spectra have been a powerful tool for studying dust in PN and related objects. Volk and Cohen (1990) were able to characterize the properties of dust emission in 170 PN by using the IRAS low resolution database. Extended red emission due to dust was discovered in bright PN by Furton and Witt (1992). Polarization studies (Johnson and Jones 1991), are proving to be a valuable tool in relating the characteristics of PN dust shells to those of related objects like Miras, $\mathrm{OH} / \mathrm{IR}$ stars and carbon stars.

\section{Shell Velocities, Morphologies and Masses}

PN shell expansion velocities are typically from about $10-100 \mathrm{~km} \mathrm{~s}^{-1}$ for the bulk of the material. However, much higher velocities in some regions have been found for objects such as He2-111 Meaburn and Walsh (1989) and NGC7139 (Walton et al 1990).

Regarding morphologies, there have been many CCD images produced of PN and a staggering amount of theory generated to explain their shapes (Frank et al 1990, Soker 1992). It remains to be decided whether stellar winds, binary nuclei or a combination of both are responsible for the shapes of PN.

Very large and faint PN halos are more common than previously believed. Thanks to the large dynamic range of CCD's, the physical properties of large halos can be studied. The masses derived for these halos are impressively large. Manchado and Pottasch (1989) find that the outer halo of NGC6543 has a mass of 1.1 solar masses, whereas the bright PN shell has only 0.07 solar masses.

\section{Central Stars}

There are now many more high quality observations of central star magnitudes than there were five years ago, particularly for PN observable in the southern skies (Jacoby and Kaler 1988, Shaw and Kaler 1989, Tylenda, et al 1989). Temperatures derived from the Zanstra and energy balance method range from 25,000 to 200,000 K (Jacoby and Kaler 1988, Preite-Martinez et al 1991).

A new view of central star spectra and magnitudes is provided by $\mathrm{x}$-ray observations (Apparao and Tarafdar 1989).

\section{Conclusions}

Much of the progress in understanding PN and their relationship to other types of objects has come from expanding the observational database. Advances in CCD's, infrared arrays and millimeter-wave technology have made possible studies that could not have been done five years ago. As has been the case so many times, the new data suggest as many problems and avenues of inquiry as they overcome.

\section{References}

Apparao, K.M.V. and Tarafdar, S.P.: 1989, Astrophys. J., 344, 826.

Dopita, M.A., Henry, J.P., Tuohy, I.R., Webster, B.L., Roberts, E.H., Byun, Y.-I., Cowie, L.L., and Sungaila, A.: 1990, Astrophys. J., 365, 640.

Frank, A., Balick, B., and Riley, J.: 1990, Astron. J., 100, 1903. 
Furton, D.G. and Witt, A.N.: 1992, Astrophys. J., 386,587.

Gomez, Y., Rodriguez, L., and Garay, G: 1992 Astron. Astrophys., 258,469.

Healey, A.P. and Huggins, P.J.: 1990, Astron. J., 100, 511.

Henry, R.B.C.: 1990, Astrophys. J., 356, 229.

Huggins, P.J. and Healey, A.P.: 1989, Astrophys. J., 98, 1662.

Jacoby, G.H. and Kaler, J.B.: 1988, Astron. J., 98, 1662.

Johnson, J.J. and Jones, T.J.: 1991, Astron. J., 101, 1735.

Kaler, J.B.: 1990, Astrophys. J., 359, 392.

Kaler, J.B. and Jacoby, G.H.: 1989, Astrophys. J., 345, 871.

Manchado, A. and Pottasch, S.R.: 1989, Astron. Astrophys., 222, 219.

Martin-Pintado, J. and Bachiller, R.: 1992, Astrophys. J. Lett., 391, 693.

Masson, C.R.: 1989, Astrophys. J., 336, 294.

Meaburn, J. and Walsh, J.R.: 1989, Astron. Astrophys., 223, 277.

Preite-Martinez, A., Acker, A., Koppen, J., and Stenholm, B.: 1991, Astron. Astrophys. Suppl., $88,121$.

Ratag, M.A., Pottasch, S.R., Dennefeld, M., and Menzies, J.W.: 1992, Astron. Astrophys., 255, 255.

Rowlands, N., Houck, J.R., Herter, T., Gull, G.E., and Skrutskie, M.F.: 1989, Astrophys. J., 341, 901.

Seaquist, E.R.: 1991, Astron. J., 101, 2141.

Shaw, R.A. and Kaler, J.B.: 1989, Astrophys. J. Suppl., 69, 495.

Soker, N.: 1992, Astrophys. J., 389, 628.

Stanghellini, L. and Kaler, J.B.: 1989, Astrophys. J., 343, 811.

Tylenda, R., Acker, A., Gleizes, F., and Stenholm, B.: 1989, Astron. Astrophys. Suppl., 77, 39.

Volk, K. and Cohen, M.: 1990, Astron. J., 100, 485.

Walton, N.A., Walsh, J.R., and Sahu, K.C.: 1990, Astron. Astrophys., 230, 445.

Webster, B.L.: 1988, Monthly Notices Roy. Astron. Soc., $230,377$.

Zahng, C.Y. and Kwok, S.: 1991, Astron. Astrophys., 250, 179.

Zijlstra, A.A. and Pottasch, S.R.: 1991, Astron. Astrophys., 243, 478. 